\title{
A CANCER PATIENT RECOMMENDER SYSTEM FOR MANAGING END-OF-LIFE DECISION MAKING
}

\author{
Raghav Pavan Karumur \\ University of Minnesota, Twin Cities, MN, USA 55455
}

\begin{abstract}
Cancer is the leading cause of death across the world. Most cancer patients get diagnosed in advanced stages, and in most cases, there is no full cure. Once diagnosed, cancer patients suffer from a variety of disorders that can lead to physical, mental, and emotional trauma. In most cases, the patient symptoms are not adequately addressed. Moreover, today's technology does not allow oncologists and clinicians to accurately estimate the patient survival time. In most countries, the end-of-life care expenditure can also be significantly high. However, research shows that palliative care and hospice services offered early in the disease trajectory can significantly bring down end-of-life care expenditures and improve the quality of care. This paper presents the design of a Cancer patient end-of-life care recommender system and primarily focuses on its various components. Using the lens of one of my studies performed in the context of Ovarian Cancer patients, in this paper, I try to establish the usefulness of such a recommender system's various components in light of other contemporary studies that have shown success using these individual tools.
\end{abstract}

\section{KEYWORDS}

Decision-Support, Recommender System, Web Intervention, Cancer

\section{INTRODUCTION}

Prior research and the World Health Organization (WHO) statistics from 2019 show Cancer as the first or second leading cause of death among people, and an important barrier in improving the life expectancy of people across the world. A GLOBOCAN report for 36 different types of cancers in 185 countries worldwide estimates 10 million cancer deaths worldwide and 19.3 million new cases in 2020 (Bray, 2020). Assuming the national rates estimated remain constant for the various countries, by 2040, the number of cancer-related deaths is expected to rise to 16.4 million, and the number of new cases is expected to rise to 29.5 million (National Cancer Institute, 2020). And cancer equally affects people of all genders. For most types of cancers, the precise causes are currently unknown and often, their symptoms are vague. Most get diagnosed at an advanced stage. There is a high risk for recurrence and in most cases of recurrence, the patients cannot be completely cured.

In the United States alone, the number of cancer-related deaths is estimated to be at 0.6 million people and the number of new diagnoses is expected to be at 1.8 million for 2020 . The number of cancer survivors was estimated to be around 16.9 million by the end of 2018 . The number is projected to be at 22.2 million by 2030 .

End-of-life care is 10-12\% of all national annual healthcare expenditures and about $25-27 \%$ of Medicare expenditures are spent on patients during the last two months of their life (Emanuel, 1996; Riley, 1993; Lubitz, 1993). In 2018, these national expenditures associated with cancer treatment and end-of-life care were estimated to be at $\$ 150.8$ billion (National Cancer Institute, 2020). In addition, significant psychological burden and emotional distress are associated with cancer diagnosis (Arriba, 2010) and these are known to persist beyond diagnosis and treatment with anywhere between $35 \%$ to $60 \%$ of the patients experiencing distress after diagnosis (Mitchell et al, 2011).

Some studies have shown that palliative care and hospice services offered early in the disease trajectory can improve the quality of care and significantly bring down the end-of-life care expenditures. Some researchers found a significant cost difference between cancer patients treated aggressively and those that sought supportive care such as hospice. The mean cost savings per physician per patient ranged from $\$ 34,677$ to $\$ 112,707$ (the range being a result of differences in individual practice patterns). Additionally, some researchers found that patients not enrolled in hospice were more likely to undergo an invasive procedure 
within a month of their closing moments. Although there are benefits associated with supportive care, most patients are not aware of such services until the fag end of their life. For instance, for 2008, the National Hospice and Palliative Care Organization (NHPCO, 2010) estimates that the median length of service was only three weeks, which means that half of the hospice patients received end-of-life care only for about three weeks!

Palliative care involves addressing physical, intellectual, emotional, social, and spiritual needs related to an illness. It also facilitates patient autonomy, access to information, and choice thereby improving the quality of life. This kind of care is provided by a multidisciplinary team made up of medical practitioners, nurses, social workers, and spiritual counselors, and is, often, offered in conjunction with all other appropriate forms of medical treatment. Cancer patients have significant physical, psychosocial, and spiritual needs for holistic care at a manageable expense from diagnosis through survivorship and end-of-life care. (CMS, 2008; WHO, 2002) This being the case, palliative care is well-positioned to address most of the symptoms that cancer patients experience throughout their disease and supports them in their journey. However, because few physicians have the adequate communication skills needed to discuss palliative care and end of life issues, expert palliative care is not currently a standard of care in treatment protocol and is not used early enough. While there is anecdotal and growing research support for the value of palliative care, evidence demonstrating sound methodology and definitive results is scarce (Petzel et al., 2009).

Thus, there are gaps in the current health care system regarding the available information and supportive resources throughout the cancer disease trajectory. The net effect of a diagnosis of cancer is that in most patients this disease has drastically poor outcomes and will result in death. For patients and family, ultimately decisions will be made surrounding end-of-life care. Despite the goal of palliative care and hospice to provide compassionate, holistic care for patients, and their families and to maximize their quality of life, most patients do not receive palliative care or hospice information until they are close to death. This approach to the end of life and the important decisions that surround this time is inadequate often making patients and their family members feel abandoned, frightened or unsure about the decisions they have to make.

This paper is based on a study that was conducted to improve end-of-life cancer management through technology. Patients and caregivers were provided with an interactive, innovative health aware recommender system (Schäfer et al., 2017) that allows access to information and decision support earlier in the disease trajectory. They were presented with information on palliative care and hospice at the time of diagnosis and at the time of their first recurrence, much earlier than this information is usually presented to them. This approach differs from the status quo in that it promotes interdisciplinary, holistic, less-expensive care for cancer patients and involves them and their caregivers much earlier in the clinical decision-making process surrounding end of life issues. This research is aimed at advancing the field of palliative care and oncology as the greater understanding of options will improve outcomes and reduce costs and thereby drastically reduce the burden of cancer on patients, families and society.

To control and tease out the effects, the study was limited to one population of Cancer patients suffering from Ovarian Cancer. The current paper primarily focuses on the design aspects of the cancer-related recommender system for making end-of-life decision making in Ovarian cancer patients. While there is already prior published work related to this stream of research (McClellan et al 2016), this paper primarily focuses on the design and the various components of the recommender system for the benefit of researchers.

\section{BACKGROUND}

Ovarian Cancer is the second most common cause of death from cancer in women in the United States (Center for Disease Control, 2021). It is one of the most pernicious forms of cancer with the highest incidence in Africa, Europe, and North America. About $70 \%$ get diagnosed at an advanced stage (Stage III-IV). The estimated five-year survival is $45 \%$ for all stages of disease, and this becomes as low as $18 \%$ for Stage IV (American Cancer Society, 2008). There is a 60-70\% risk for recurrence and women who recur currently cannot be cured.

Symptoms such as abnormal menstrual cycles, and digestive symptoms such as constipation, increased bloating, indigestion, lack of appetite, nausea and vomiting, unexplained back pain that worsens over time, vaginal bleeding between periods, unusual weight gain or loss, excessive hair growth that is coarse and dark, sudden urge to urinate, and needing to urinate more often than usual may be indications of ovarian cancer. Because these symptoms are also common in women who do not have ovarian cancer, the majority of cases are diagnosed late. By the time the cancer is diagnosed, the tumor often spreads beyond the ovaries. 
Also, no lab or imaging test has ever been shown to be able to screen for or diagnose ovarian cancer in its early stages. A physical examination or surgery such as a pelvic laparoscopy or exploratory laparotomy, ultrasound, $\mathrm{X}$-rays, the CA-125 blood test may be done to evaluate symptoms and perform a biopsy to help make the diagnosis and determine staging. Only about $20 \%$ of the women are diagnosed early, when the disease may be curable.

Patients with ovarian cancer are commonly impacted by symptom clusters including but not limited to fatigue, anxiety, pain, bloating, bowel disturbances, nausea and vomiting, anorexia, and depression (Bankhead et al. 2005). Long time effects of survivors include surgical menopause, gender and role identity disturbances, peripheral neuropathy, cognitive dysfunction, and decreased quality of life related to fears of recurrence, sexual dysfunction, depression, and employability and symptoms are undertreated in 16-49\% of survivors (Koldjeski, 2007; Le, 2003, 2005; Lockwood-Rayermann, 2006). In a study by Stone et al. (2000), more than half of the participants ranked cancer-related fatigue worse than anxiety, pain, and nausea/vomiting. However, in 59\% of them, cancer-related fatigue goes unreported to oncologists, while 53\% never receive counseling from oncologists on how to treat fatigue (Donovan and Ward, 2005). This calls for the necessity of access to a multidisciplinary palliative care team.

Critical questions persist concerning the decision-making processes involved. For example, how women approach palliative care and hospice at different times during their disease trajectory and what might be done to assist women with ovarian cancer in making decisions surrounding palliative care and the end-of-life. This confirms the need for rigorous study of how women with ovarian cancer use palliative care and hospice information and support at different times from diagnosis through end-of-life care.

Research studies have shown that about $80 \%$ of adult web users have searched online for health information and seniors on the internet are the fastest growing internet population. Most Ovarian cancer patients want to be aware, informed and are in need of constant social support. In general, they suffer from loss of memory, difficulty in remembering, anxiety. They look for similar people to connect and share their thoughts. So, an online forum serves this purpose the best.

Also, most existing websites either present heavy text-based information, or information that is not timed to the patient's stage of disease or emotional state at the time they access the information, and lack proper discussion forums, or existing forums are not well-maintained. Many questions never get answered, and some of them present conflicting information because of lack of moderators.

Given these constraints, an online portal was designed for Cancer patients that provides holistic care and supports the end-of-life decision-making in collaboration with oncologists from Fairview Masonic Cancer Clinic, psychologists, decision support system experts, and usability experts. The portal was designed with interactive controls and resources tailored to the phase of the disease. The portal comprised of multiple modules such as a content management system, a social networking platform, a scheduling system, and distress monitoring system. This paper is primarily focused on describing the various components of this portal.

The objective was to design and pilot-test a technology-based approach for recurrent ovarian cancer patients, families, and their providers to assist in and study the decision-making process that surrounds end-of-life decisions, specifically things such as entry into hospice care. The central scientific hypothesis is that an interactive web-based system designed with and for patients, families, and providers that can provide support and tailor information to women's needs at the end-of-life will increase the number of patients electing to use hospice care and at a time point earlier in the course of their disease and will result in improved outcomes for patients and their families in terms of better quality of life.

Women with advanced stage ovarian cancer at the time of diagnosis and at recurrence were planned to be recruited and a pilot evaluation study was designed using a longitudinal, before, during, and after, experimental design with two arms - a control group and an intervention group. All subjects were to complete a battery of demographic, psychosocial, health status and outcomes, health information, and decision-making measures at baseline and 30-day post-baseline. Primary patient data was planned to be complemented with continuous data automatically collected by the system as well as clinical data from medical records. In order to develop useful guidelines for these discussions, reliable data about the prognostic indicators and an understanding of the psychological factors surrounding patient preferences and decisions are necessary.

The intervention group was to be presented with a wide gamut of resources and tools in a customized way while the control group will have access to all the documents presented to the intervention group, but with no customization. The study was aimed primarily at understanding how the behavior of patients exposed to controlled and customized content varies from those that are presented with random content. Therefore, I have developed two different portals - one for the control group patients with the least amount of customization, 
and the other for the intervention group patients with maximum amount of customization. Along with these, I have developed two other portals - one for the patient's caregiver and one for the clinician whom the patient visits. While the caregiver has a portal similar to that of the intervention group patient whom they are caregiving, both the caregiver as well as the clinician are presented with a summary view of how the patient's end-of-life behavior is changing as is being intervened by the currently designed portal.

\section{IMPLEMENTATION}

\subsection{Key Elements of the Recommender System}

The system was originally envisioned to have a look and feel similar to popular social networking websites and blogs. This would mean including features that allow users to create and modify their own profile, connect with peers, and post to discussion forms. Profile fields were seeded to encourage participants to address specific issues of living with ovarian cancer. In addition to social networking features, core intervention components such as medication, appointment, and symptom monitoring would be made available, providing tailored feedback on disease and psychological health.

Our aim was to design a creative user interface that is intuitive and optimized for patients at every level/stage of illness. During the design events, we have learned that the patients needed an all-the-time access to their families, caregivers and healthcare team during their end-of-life. This motivated us to bring the three categories of people together, having three subdivisions within our portal - one for patients, one for their caregivers and one for the physicians. While each of them has space for their own journaling and participation in forum discussions, they also can mutually interact with one another.

Many patients have indicated that they wanted a way to easily keep track of past appointment dates with summaries, current and upcoming appointments. This has motivated us to provide access to a calendar, that they can use to quickly browse through their appointments.

Studies on cancer patients found that $62.5 \%$ preferred participating in clinical decisions (Cassileth BR, et al., 1980) and that younger patients were significantly more likely to desire participation that were patients over age 60. Other studies however found that in the context of cancer, a majority of patients preferred their physician to make treatment decisions on their behalf (Degner and Sloan, 1992; Sutherland, 1989). Still another study of breast cancer patients found that two thirds preferred autonomous or collaborative treatment decision making (Degner, 1997). In order to make the decision-making process easier, we have provided a section called "Action Plan" which facilitates the users to maintain a quick list of items they need to make decisions on.

Critical questions persist concerning the decision-making processes involved. For example, how women actually approach palliative care and hospice at different times during their disease trajectory and what might be done to assist women with ovarian cancer in making decisions surrounding palliative care and the end-of-life. Therefore, we believe that the data collected from these action plan items could help us better understand the decision-making process surrounding palliative care and the end-of-life.

According to Zimmermann et al., (2008), cancer patients have significant physical, psychosocial, and spiritual needs for palliative care from "diagnosis through survivorship and end-of-life care". To address this, we have provided access to a rich library of topics and resources called "Learning Library" categorized into eight major families - (1) Palliative Care, (2) Decision Making (3) Understanding Ovarian Cancer, (4) Practical Considerations, (5) Emotional Well-being, (6) Physical Well-being, (7) Social Support, and (8) Communication.

Furthermore, a selected list of topics relevant to the patient based on their stage of disease is presented in the "Recommended topics" section. We have basically identified four categories of patients, namely - newly diagnosed, recurrent, multiply recurrent and remission. Each is presented with their own set of recommended topics.

Often times cancer patients are unable to speak for themselves due to sudden medical crisis. Hence, they often choose to nominate a person to make healthcare decisions on their behalf in case they become unable to make or communicate their decisions. These nominations are usually done using a form called "Advance Healthcare directive". We have included this along with the instructions to fill this out in our website. 
A distress thermometer is a validated tool used to monitor one's psychological distress level. Use of this tool is important in cancer related distress as often this is not recognized by cancer care providers, although it is present in the patients. The drawback of this is that when it is not recognized, it is likely that interventions known to reduce their distress will not occur. And increased distress is related to several negative outcomes for patients, including poorer satisfaction with care, lower follow through with treatment recommendations and poorer quality of life. Therefore, we have provided a distress thermometer for the patients to periodically record their distress levels along with the factors contributing to the distress. There is also a provision for the patients to let their providers and caregivers view their distress levels and factors using a stress graph.

Also provided in this portal are sections for patients to vent their feelings and thoughts through social networking. Journaling is an expressive way of writing very personally about emotional and/or stressful experiences and research demonstrates that it can have beneficial effects on emotional well-being and physical wealth. Writing about an upsetting event such as cancer can limit its impact in several ways by (a) providing a new understanding of cancer experience to the patients. (b) helping them limit possible threatening thoughts or images (c) encouraging positive expectations (d) improving sleep and increasing functioning during the day in case of some advance-disease cancer patients and so on. It is often times helpful to write about one's positive responses to illness, such as finding benefit in their cancer experience. Hence, we have provided active ways for patients to journal and communicate with their caregivers.

Ovarian cancer patients (cancer patients in general) look for solace in the form of support groups and forums to exchange ideas with similar women or those that have similar experiences. Research shows that based on common experiences, women who discuss may help others understand their illness differently and how to combat against it. It also helps them understand in ways that friends, family and providers haven't provided. Hence, we have provided discussion forums that represent using social media to share, interact and communicate with similar groups of women having similar experiences.

Clinical trials are research studies focused on finding ways to improve health and cancer care. Each study tries to answer scientific questions relating to the prevention, diagnosis, or treatment cancer. We have also included a section on clinical trials along with suitable decision aids regarding clinical trials.

Some of the key components are listed below

Calendar A calendar has been provided to the patients for easily keep track of past appointment dates with summaries, current and upcoming appointments. They can enter their medical appointments as well as other important appointments.

Action Plan It is a place for the patients to have their own to-do items which can be added from within various topics by clicking on one or more of the suggested action plan items as shown below:

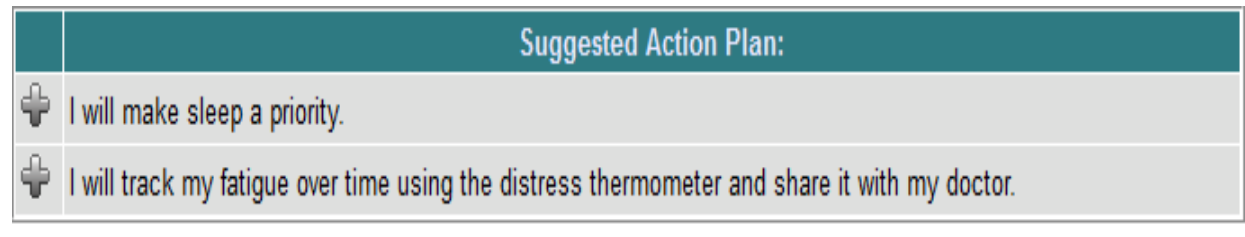

Figure 1. A snapshot of the Patient's Suggested Action Plan

Learning Library A place where the patients have a list of recommended readings for them based on their stage of disease, followed by the full library of educational topics. A quick list of this is also provided on the patient home page as shown below: 


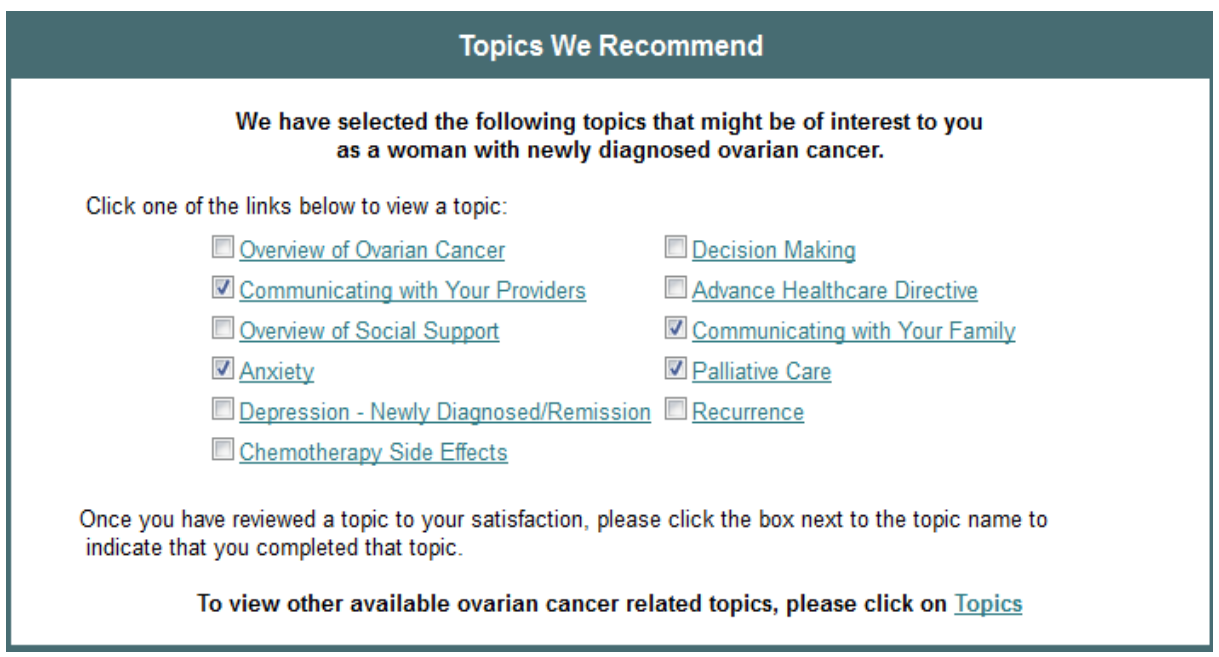

Figure 2. A snapshot of the recommended topics to the patients

Distress Thermometer This is a place where the patients can use a thermometer to enter and track their levels of distress over time. Along with the thermometer, the patients are presented with a list of factors they can choose from, that have contributed to their distress in the past week including that day. They also have an option to view a summarized list of factors that contributed to distress, along with a graph depicting their distress levels during the course of the study. On the other hand, both the caregiver and the clinician are presented with a summary view of how the patient's end-of-life behavior is changing as is being intervened by our portal.

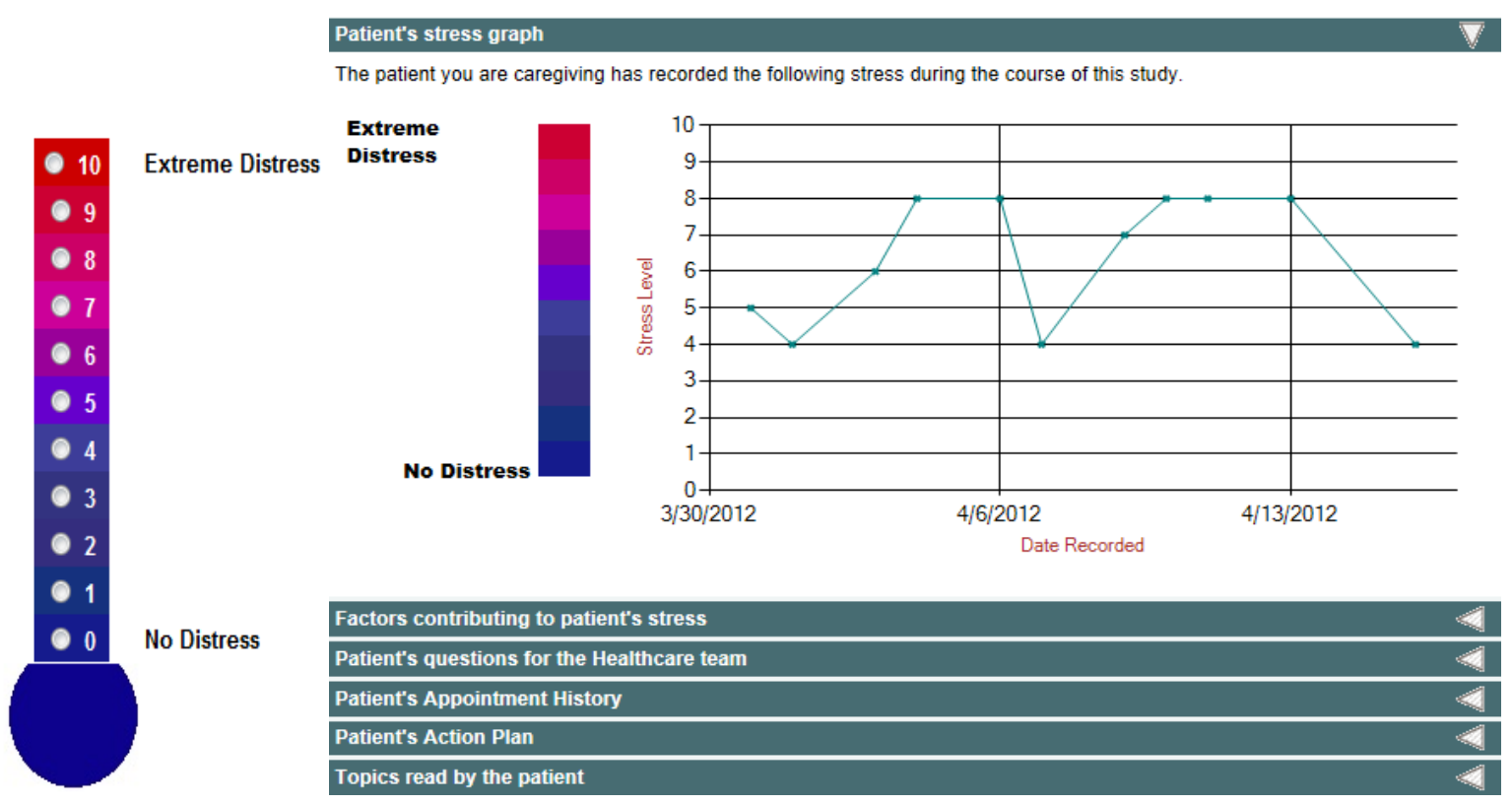

Figure 3. Snapshots of the Patient's Distress Thermometer and the Distress Graph

Journal and Discussion Forum The journal is a place where the patients can write about how they are feeling. They have an option to keep it private or share it with their caregiver. The Discussion Forum is a public avenue to post questions and share thoughts with other woman having ovarian cancer. 


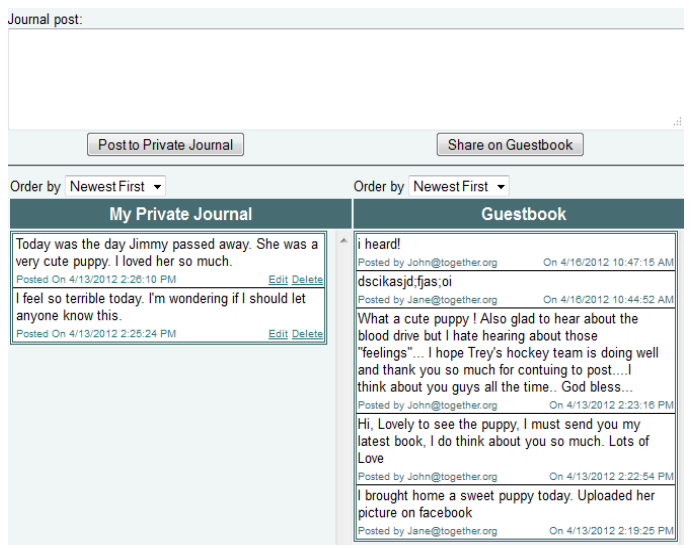

\begin{tabular}{|c|c|c|c|}
\hline \multirow{3}{*}{$\begin{array}{l}\text { Subject } \\
\text { Post: }\end{array}$} & \multicolumn{3}{|c|}{ 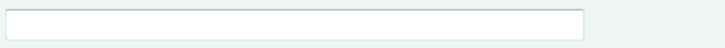 } \\
\hline & \\
\hline & \multicolumn{3}{|l|}{ Cancel } \\
\hline \multicolumn{2}{|c|}{ Order by NewestFirst $\mathbf{v}$} & & Search \\
\hline & Subject & |Replies & Last Update \\
\hline $\begin{array}{l}\text { g-tube } \\
\text { Created by Jane }\end{array}$ & gogetherorg & 1 & 4//6/2012 10:48:31 AM \\
\hline $\begin{array}{l}\text { How fast do } \\
\text { Crested by Suss }\end{array}$ & $\begin{array}{l}\text { arian cysts/cancer grow? } \\
\text { patientcoom }\end{array}$ & 2 & 4/13/2012 2:15:15 PM \\
\hline $\begin{array}{l}\text { side effects } \\
\text { Creeted by marts }\end{array}$ & chemo & 3 & 4/13/2012 2:16:45 PM \\
\hline 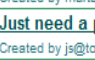 & ace to voice my fears & 3 & 3/200/2012 12:25:22 PN \\
\hline $\begin{array}{l}\text { Is this a com } \\
\text { Created by Susar }\end{array}$ & $\begin{array}{l}\text { on symptom of ovarian cancer? } \\
\text { patientcom }\end{array}$ & 2 & 3/115/2012 10:38:21 AN \\
\hline
\end{tabular}

Figure 4. Snapshots of the Patient's Journal and Discussion Forum

Glossary This is an index of terms and definitions found in this website

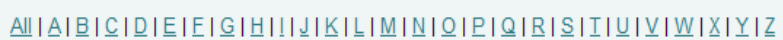

\begin{tabular}{|ll||}
\hline IERM & DEFINITION \\
benign tumor & $\begin{array}{l}\text { A tumor which is non-cancerous. These generally grow slowly and do not invade adjacent organs or } \\
\text { spread (metastasize) beyond the ovaries. }\end{array}$ \\
\hline biliary tree & Branched bile ducts much like a tree \\
\hline biomarker & A distinctive usually biochemical indicator of a biological or geochemical process or event \\
\hline biopsy & The removal and microscopic examination of a tissue sample. \\
\hline $\begin{array}{l}\text { borderline } \\
\text { tumor }\end{array}$ & $\begin{array}{l}\text { Borderline (low malignant potential (LMP)) tumors are a borderline form of cancer that may eventually } \\
\text { spread and invade other tissues. This is a gray zone. }\end{array}$ \\
\hline
\end{tabular}

Question for my Healthcare Team:

Please type your question here to add it to your list.

Add

View all questions

Figure 5. A snapshot of the Patient's Glossary list

\subsubsection{Healthcare Team and Questions for the Healthcare Team}

This contains access to the multidisciplinary palliative care team made up of medical practitioners, nurses, social workers, spiritual counselors. The Questions section can be used by the patients to write down questions they always wanted to ask their providers.

\section{CONCLUSION}

To address the challenges associated with end-of-life care such as insufficiently addressed symptoms, lack of supportive care for a significant portion, and substantially high expenditures, an end-of-life cancer recommender system was designed to support patients through their cancer journey. This paper is part of a stream of research publications which focuses only on the elements of the design of the recommender system. For the details related to the usability testing of this tool, the readers of this work are requested to refer to McClellan, M.A (2016).

\section{ACKNOWLEDGEMENT}

I would like to acknowledge the gynecological oncology team at the University of Minnesota that assisted me with my development of this portal and funded my research through a grant from the Academic Health Center. 


\section{REFERENCES}

American Cancer Society. Cancer Facts and Figures 2008. Atlanta: American Cancer Society; 2008.

Arriba, L.N., 2010. A review of issues surrounding quality of life among women with ovarian cancer. Gynecologic oncology, 119(2), pp.390-396.

Bankhead et al. 2005. Symptoms associated with diagnosis of ovarian cancer: a systematic review. BJOG, 112; 857-865.

Bray, F., 2020. Erratum: Global cancer statistics 2018: GLOBOCAN estimates of incidence and mortality worldwide for 36 cancers in 185 countries. CA Cancer J Clin, 70(4), p.313.

Cassileth BR, et al. 1980. Information and participation preferences among cancer patients. Ann Intern Med. 1980; 92: 832-836.

Center for Disease Control, 2021. https://www.cdc.gov/cancer/ovarian/statistics/index.htm

Centers for Medicare \& Medicaid Services (CMS) 2008, p.2204. Center to Advance Palliative Care. Defining palliative care. Retrieved Dec 23, 2008, from http://www.capc.org/building-a-hospital-based-palliative-careprogram/case/definingpc/

Degner LF and J Sloan. 1992. Decision making during serious illness: What role do patients want to play? J Clin Epidemiol 45, pp. 941-950.

Degner LF, et al. 1997. Information needs and decisional preferences in women with breast cancer. JAMA; 277(18): $1485-1492$

Donovan, H.S., \& Ward, S. 2005. Representations of fatigue in women receiving chemotherapy for gynecologic cancers. Oncology Nursing Forum, 32, 113-116. doi: 10.1188/05.ONF.113-116

Emanuel EJ. 1996. Cost savings at the end of life. What do the data show? Jama ;275: 1907-14.

Koldjeski, D et al. 2007. Families coping with cancer. Cancer Nursing,

Le, $\mathrm{T}$ et al. 2005. Quality of life assessments in epithelial ovarian cancer patients during and after chemotherapy. International Journal of Gynecological Cancer, 15, 811-816.

Le, $T$ et al. 2003. Quality-of-life issues in patients with ovarian cancer and their caregivers: A review. Obstetrical and Gynecological Survey, 58(11), 749-758.

Lockwood-Rayermann, S. 2006. Survivorship issues in ovarian cancer: A review. Oncology Nursing Forum, 33(3), 553-562.

Lubitz JD, Riley GF. 1993. Trends in Medicare payments in the last year of life. N Engl J Med;328: 1092-6.

Stone, P., et al. 2000. Cancer- related fatigue: inevitable, unimportant and untreatable? Results of a multi-center patient survey. Cancer Fatigue Forum Annals of Oncology 11: 8, 971-975.

McClellan, M.A., et al. 2016. Designing an educational website to improve quality of supportive oncology care for women with ovarian cancer: an expert usability review and analysis. International journal of human-computer interaction, 32(4), pp.297-307.

Mitchell, A. J., et al. 2011. Prevalence of depression, anxiety, and adjustment disorder in oncological, hematological, and palliative-care settings: a meta-analysis of 94 interview-based studies. The lancet oncology, 12(2), 160-174.

National Cancer Institute. Cancer Statistics 2020. https://www.cancer.gov/about-cancer/understanding/statistics

NHPCO, www.nhpco.org/nds, accessed online on March 9, 2010.

Ovarian Cancer, A.D.A.M Medical Encyclopedia, U.S. National Library of Medicine

Petzel S et al. Learning about cancer: Pros and cons. Psychosomatic Medicine. 2009;71(3): A30.

Schäfer, H., et al. 2017. Towards health (aware) recommender systems. In Proceedings of the 2017 international conference on digital health (pp. 157-161).

World Health Organization 2002. WHO definition of palliative care. Retrieved April 13, 2008 from http://www.who.int/cancer/palliative/definition/en/

Zimmermann, C, et al. Effectiveness of specialized palliative care: A systematic review. Journal of the American Medical Association. 2008;299(14):1698-1709. 